\title{
Minireview Proton beam therapy
}

\author{
WP Levin', H Kooy', JS Loeffler' and TF DeLaney*, \\ 'Massachusetts General Hospital Northeast Proton Therapy Center, Boston, MA 02 I I4, USA
}

\begin{abstract}
Conventional radiation therapy directs photons (X-rays) and electrons at tumours with the intent of eradicating the neoplastic tissue while preserving adjacent normal tissue. Radiation-induced damage to healthy tissue and second malignancies are always a concern, however, when administering radiation. Proton beam radiotherapy, one form of charged particle therapy, allows for excellent dose distributions, with the added benefit of no exit dose. These characteristics make this form of radiotherapy an excellent choice for the treatment of tumours located next to critical structures such as the spinal cord, eyes, and brain, as well as for paediatric malignancies. British Journal of Cancer (2005) 93, 849-854. doi:I0.1038/sj.bjc.6602754 www.bjcancer.com
\end{abstract}

Published online 27 September 2005

(c) 2005 Cancer Research UK

Keywords: radiotherapy; protein beam

Conventional radiation therapy, which utilises photon (X-ray) beams, is frequently used in the locoregional treatment of cancer. Tumour control is achieved by radiation-induced damage to DNA, which ultimately causes tumour cell death. In vitro, even the most radioresistant cancers can be eliminated. In vivo, however, lethal tumour doses are not always achievable because of radiationinduced morbidity in normal tissues.

Radiation is currently delivered with substantially more precision than in the past because of advances in imaging and treatment planning. To date, the most advanced photon beam delivery method is intensity-modulated (IM) radiation therapy (IMRT), which can deliver higher doses of radiotherapy to tumour targets while reducing the dose delivered to selected normal tissues. With IMRT, high doses to these selected normal tissues can be avoided by applying numerous radiation fields of varying intensities from different directions. But this requires increasing the volume of normal tissue that is irradiated (i.e. a higher integral dose); hence, one of the concerns of IMRT is that, over time, this exposure of more tissue to low-dose radiation will cause a second malignancy or other unwanted late normal tissue effect. This is especially concerning with regards to paediatric patients receiving IMRT (Miralbell et al, 2002). If these children are cured of their primary cancer, they should have a relatively long lifespan, during which time they may manifest a radiation-induced malignancy. Of course, the risk of second malignancy is also a risk in adults, but the fact that the median age of adult cancer patients at diagnosis is in the seventh decade, that second malignancies after radiotherapy are uncommon in adults, and that they usually manifest 10-15 years after treatment make them less of a concern. It is also worth mentioning that intensity modulation using a scanned pencil beam has also been applied to proton radiotherapy; dose distributions are superior to those achievable with the more commonly employed passively scattered proton beams (Weber et al, 2004),

\footnotetext{
*Correspondence: Dr TF DeLaney; E-mail: TDELANEY@PARTNERS.ORG

Received 12 May 2005; revised 29 July 2005; accepted 29 July 2005; published online 27 September 2005
}

and there is also less total body neutron dose from beam-shaping devices (Miralbell et al, 2002).

Interest in the use of charged particle radiotherapy has been primarily stimulated by the superior dose distributions - already recognised by Wilson (1946) - compared to those produced by photon therapy techniques. Protons, as do all charged particles, have a very rapid energy loss in the last few millimeters of penetration. This results in a sharply localised peak of dose, known as the Bragg peak. The penetration depth of the Bragg peak is directly related to the initial energy of the charged particle. The Bragg peak, and hence desired dose, can thus be precisely placed anywhere in the patient (see Figure 1). For irradiation of a tumour, the proton beam energy and intensity are varied in order to achieve the desired dose over the tumour volume. A single clinical proton field, in contrast to a single photon field, can achieve dose conformation to the target volume. In general, a set of proton fields achieves significant dose reduction to uninvolved normal tissues compared to a matched set of photon fields. Passively scattered proton fields have a slightly higher entrance dose at the skin ( $\sim 75 \%)$ compared to megavoltage photon beams ( $\sim 60 \%)$; more than one port may be required with protons if adequate skin sparing is to be achieved in patients being treated to high doses with only protons.

Protons have comparable biologic effects in tissue relative to high-energy X-rays used in conventional radiation therapy. Evidence of this comes from the fact that the relative biological effectiveness (RBE) of protons is approximately 1.1 (Paganetti et al, 2002). The RBE of a proton beam is the ratio of the dose required to produce a specified effect using a reference radiation, usually ${ }^{60} \mathrm{Co}$ photons, to the dose required to produce the same effect. A generic RBE factor of 1.1 has been used at the Harvard Cyclotron, Northeast Proton Therapy Center, Loma Linda University, Paul Scherrer Institute in Switzerland, Orsay in France, and Faure in South Africa, while Tsukuba in Japan and Uppsala in Sweden have used 1.0. It is important to contrast these biologic and physical properties of protons with those of neutrons and heavier charged particles. Fast neutrons have a higher RBE in tumour cells, but lack the physical dose advantages of charged particles; the latter proved disadvantageous in the clinic 


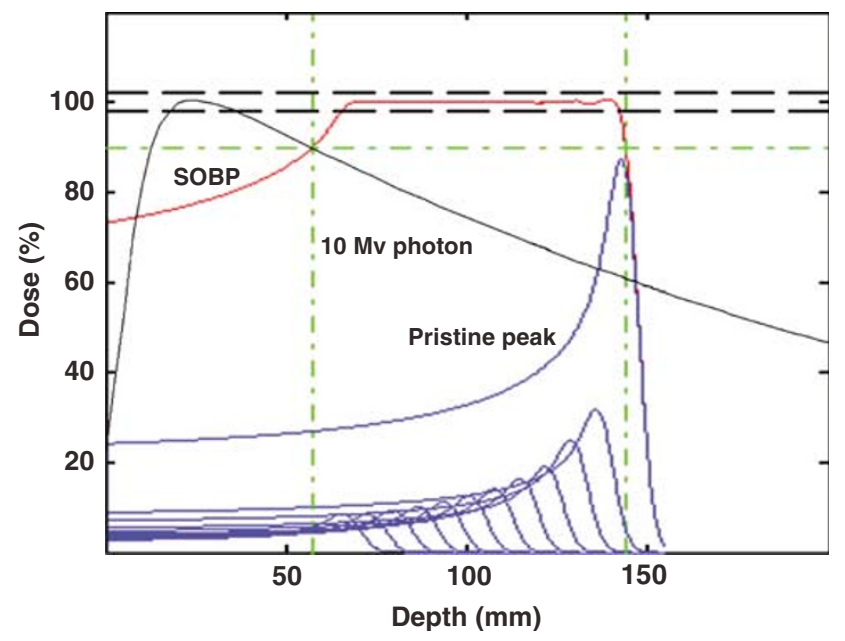

Figure I Depth-dose distributions for a spread-out Bragg peak (SOBP, red), its constituent pristine Bragg peaks (blue), and a IO MV photon beam (black). The SOBP dose distribution is created by adding the contributions of individually modulated pristine Bragg peaks. The penetration depth, or range, measured as the depth of the distal $90 \%$ of plateau dose, of the SOBP dose distribution is determined by the range of the most distal pristine peak (labeled 'Pristine peak'). The modulation width, measured as the distance between the proximal and distal $90 \%$ of plateau dose values, of the SOBP dose distribution is controlled by varying the number and intensity of pristine Bragg peaks that are added, relative to the most distal pristine peak, to form the SOBP. The dashed lines (black) indicate the clinical acceptable variation in the plateau dose of $\pm 2 \%$. The dot-dashed lines (green) indicate the $90 \%$ dose and spatial, range and modulation width, intervals. The SOBP dose distribution of even a single field can provide complete target volume coverage in depth and lateral dimensions, in sharp contrast to a single photon dose distribution; only a composite set of photon fields can deliver a clinical target dose distribution. Note the absence of dose beyond the distal fall-off edge of the SOBP.

(Wambersie and Menzel, 1996). Heavier charged particles like carbon ions combine a higher RBE with an improved physical dose distribution; they may offer additional advantages for hypoxic and other radioresistant tumours and clinical studies are in progress at NIRS in Japan (Kamada et al, 2002; Tsujii et al, 2004) and GSI in Germany (Schulz-Ertner et al, 2004). Many clinical studies with protons have employed a combination of photons and protons; the combination is facilitated by the similar biologic effects.

\section{PROTON BEAM RADIOTHERAPY}

The majority of patients receiving charged particle therapy have been treated with protons. As of July 2004, over 39000 patients have received part or all of their radiation therapy (RT) by proton beams (Sisterson, 2004). Table 1 lists the currently operational proton beam treatment facilities worldwide.

Initially, patients were being treated at facilities designed and constructed for basic high-energy physics research, often resulting in very cumbersome treatments, as the proton beams were limited to a fixed (often horizontal) position, which meant that the patient had to be moved to align the tumour on the trajectory of the beam. This technique was in contrast to the isocentric capabilities of the modern linear accelerator that rotates around a point in space and can effectively target any site in the body. In addition, for many of the proton machines, the energy of the beam (which defined the depth of the Bragg peak) was only sufficient to treat superficial lesions (such as those of the eye) or intermediate-depth lesions (such as the base of skull). Owing to these technical factors and the interests of the involved physicians, the clinical sites that had
Table I Operational proton therapy centres

\begin{tabular}{llrrr}
\hline Facility & Location & $\begin{array}{c}\text { Date } \\
\text { first } \\
\text { RX }\end{array}$ & $\begin{array}{r}\text { Recent } \\
\text { patient } \\
\text { total }\end{array}$ & $\begin{array}{c}\text { Date of } \\
\text { total }\end{array}$ \\
\hline ITEP, Moscow & Russia & 1969 & 3748 & June-04 \\
St Petersburg & Russia & 1975 & 1145 & April-04 \\
Chiba & Japan & 1979 & 145 & Apr-02 \\
PSI & Switzerland & 1984 & 4066 & June-04 \\
Dubna & Russia & 1999 & 191 & Nov-03 \\
Uppsala & Sweden & 1989 & 418 & Jan-04 \\
Clatterbridge & England & 1989 & 1287 & Dec-03 \\
Loma Linda & California, USA & 1990 & 9282 & July-04 \\
Nice & France & 1991 & 2555 & April-04 \\
Orsay & France & 1991 & 2805 & Dec-03 \\
iThemba LABS & South Africa & 1993 & 446 & Dec-03 \\
UCSF - CNL & California, USA & 1994 & 632 & June-04 \\
TRIUMF & Canada & 1995 & 89 & Dec-03 \\
PSI & Switzerland & 1996 & 166 & Dec-03 \\
HMI, Berlin & Germany & 1998 & 437 & Dec-03 \\
NCC, Kashiwa & Japan & 1998 & 270 & June-04 \\
HIBMC, Hyogo & Japan & 2001 & 359 & June-04 \\
PMRC, Tsukuba & Japan & 2001 & 492 & July 04 \\
NPTC, MGH & Massachusetts, USA & 2001 & 800 & July-04 \\
INFN-LNS, Catania & Italy & 2002 & 77 & June-04 \\
WERC & Japan & 2002 & 14 & Dec-03 \\
Shizuoka & Japan & 2003 & 69 & July-04 \\
MPRI & Indiana, USA & 2004 & 21 & July-04 \\
\hline & & & &
\end{tabular}

initially received the most attention were uveal melanomas in the eye and base of skull sarcomas. The major emphasis for proton therapy clinical research initially was dose escalation for tumours adjacent to critical normal structures that constrained the doses that could be given with photons and for which local tumour control with conventional radiotherapy was thus poor. One of the pioneers in proton radiation therapy was the research facility at the Harvard Cyclotron Laboratory (HCL) in Cambridge, Massachusetts, operating in conjunction with the Massachusetts General Hospital. Patient treatment commenced in 1961 and ended in 2002, after the clinical programme was transferred to the Northeast Proton Therapy Center at Massachusetts General Hospital. In total, 9116 patients were treated at the HCL.

The development of hospital-based cyclotrons with higher energy beams capable of reaching deep-seated tumours (up to $\sim 30 \mathrm{~cm}$ ), field sizes comparable to linear accelerators, and rotational gantries have greatly facilitated proton radiation therapy. The first of these hospital-based facilities opened at Loma Linda University in California in 1990. Increasingly, there is interest in protocols aimed at morbidity reduction in those tumour sites in which tumour control with photons is good, such as many paediatric tumours.

\section{OCULAR (UVEAL) MELANOMA}

Uveal melanoma is the most common primary ocular tumour. Episcleral radioactive plaques and proton beam radiation are alternatives to enucleation with the intent of preservation of sight. The latter is not always achievable due to the proximity of the cornea, lens, retina, fovea, or optic nerve. Typically, a total of 70 Cobalt Gray Equivalent (CGE) (1 CGE represents the physical dose of protons multiplied by an RBE factor and should thus have similar biologic effects in the system of interest as 1 Gray (Gy) of photon dose) is administered over five treatment sessions.

As of December 2002, over 3000 patients with uveal melanoma had been treated with protons at the MGH in collaboration with MEEI (Munzenrider, 1999). The 5-year actuarial local control rate 
was $96 \%$ for all sites within the globe, with an $80 \%$ overall survival. The probability of eye retention at 5 years was estimated to be $90 \%$ for the entire group and 97, 93, and $78 \%$ for patients with small, intermediate, and large tumours, respectively.

Egger et al (2003) recently reported long-term results of eye retention after treatment of uveal melanoma with proton beam therapy. A total of 2645 patients were treated at Paul Scherrer Institute in Switzerland, between 1984 and 1999. The overall eye retention rates at 5, 10, and 15 years after treatment were 89,86 , and $83 \%$, respectively.

\section{SARCOMAS OF THE SKULL BASE AND SPINE}

Treatment of patients with sarcoma of the skull base is very challenging because of the proximity of critical structures, notably, the brain, brainstem, cervical cord, optic nerves, and optic chiasm. Accordingly, surgery and conventional photon therapy has not been very successful at controlling these tumours. Owing to the necessity to deliver dose in a precise manner, the use of proton therapy is becoming the treatment of choice for these tumours.

At the Harvard Cyclotron (HCL), MGH physicians used a combination of protons and photons to treat patients with tumours of the skull base and cervical spine (Munzenrider and Liebsch, 1999). A total of 169 patients with chordoma and 165 patients with chondrosarcoma were treated. Local control (10-year) for skull base tumours was highest for chondrosarcomas, intermediate for male chordomas, and lowest for female chordomas $(94,65$, and $42 \%$, respectively). For cervical spine tumours, 10 -year local control rates were not significantly different for chordomas and chondrosarcomas (54 and 48\%, respectively), nor was there any significant difference in local control between males and females. Actuarial rates (5-year) of endocrinopathy in patients with base of skull lesions were as follows: $72 \%$ for hyperprolactinaemia, $30 \%$ for hypothyroidism, $29 \%$ for hypogonadism, $19 \%$ for hypoadrenalism, and no incidence of diabetes insipidus (Pai et al, 2001), reflective of the proximity of the pituitary to the sarcoma.

Treatment of spinal and paraspinal tumours is complicated by the proximity of the spinal cord. Radiation tolerance of the spinal cord is generally quoted at $45 \mathrm{~Gy}$, well below that necessary to reliably control most sarcomas, which require doses of approximately $60 \mathrm{~Gy}$ for subclinical microscopic disease, $66 \mathrm{~Gy}$ for microscopically positive margins, and in excess of $70 \mathrm{~Gy}$ for gross residual disease. Proton radiotherapy, with its ability to spare adjacent tissues, offers advantages for treatment of tumours in this location. Hug et al (1995) presented results on combined photon/ proton treatment of 47 patients with osteo- and chondrogenic tumours of the axial skeleton. Actuarial local control (5-year) and survival for patients with chondrosarcoma were 100 and $100 \%$, and with chordoma were 53 and 50\%. Actuarial 5-year local control for patients with osteosarcoma was $59 \%$.

\section{BENIGN MENINGIOMA}

Complete surgical resection of meningiomas is difficult to achieve in selected locations such as the sphenoid ridge, parasellar area, and posterior fossa. Likewise, radiation therapy for these intracranial tumours is complicated by the proximity of critical neural structures, such as the visual pathways or the brain stem. Proton beam radiation, with its high degree of conformality, therefore would seem to be an attractive treatment modality.

Between 1981 and 1996, 46 patients with partially resected, biopsied, or recurrent benign meningiomas were treated with combined proton/photon radiation at the HCL/MGH (Wenkel et al, 2000). The median dose to the tumour was 59 CGE. Overall survivals at 5 and 10 years were 93 and $77 \%$, respectively, and the recurrence-free rates at 5 and 10 years were 100 and $88 \%$, respectively. Three patients presented with local tumour recurrence at 61,95 , and 125 months. One patient died of focal brain necrosis at 22 months. Neurologic complications, including memory deficits and hearing loss, were also seen. Four patients developed ophthalmologic toxicity. In all of these cases maximum dose to the optic structures was greater than 58 CGE. Endocrine abnormalities following treatment were also seen.

Investigators from Paul Scherrer Institute recently reported on the treatment of 16 patients with recurrent, residual, or untreated intracranial meningiomas (Weber et al, 2004). The median prescribed dose was 56 CGE (52-64) at 1.8-2 CGE per fraction. Cumulative 3-year local control, progression-free survival, and overall survival were 91,91 , and $92 \%$, respectively. No patient died of recurrent meningioma. Radiographic follow-up (median, 34 months) revealed an objective response in three patients and stable disease in 12 patients. Cumulative 3-year toxicity-free survival was $76 \%$. No radiation-induced hypothalamic/pituitary dysfunction was observed.

Encouraging data with stereotactic external beam radiotherapy and IMRT, however, have also been reported (Uy et al, 2002; Zabel et al, 2005) and comparative studies need to be conducted to assess whether there is any demonstrable difference in tumour control or complications between these techniques and proton radiotherapy.

\section{PARANASAL SINUS, NASAL, AND NASOPHARYNGEAL TUMOURS}

Fitzek et al (2002) performed a prospective study incorporating chemotherapy, surgery, and combined proton-photon radiotherapy for treatment of malignant neuroendocrine tumours of the sinonasal tract. In all, 19 patients with olfactory neuroblastoma (ONB) or neuroendocrinecarcinoma (NEC) were treated with two courses of cisplatin/etoposide chemotherapy, followed by highdose proton-photon radiotherapy to 69.2 CGE using 1.6-1.8 CGE per fraction twice daily in a concomitant boost schedule. Two further courses of chemotherapy were given to responders. The 5year survival rate was $74 \%$. The 5 -year local control rate of initial treatment was $88 \%$. Acute toxicity of chemotherapy was tolerable, with no patient sustaining more than grade 3 haematologic toxicity. One patient developed unilateral visual loss after the first course of chemotherapy; otherwise, the precision of delivery of radiation with stereotactic setup and protons resulted in visual preservation in all patients. Four patients who were clinically intact developed radiation-induced damage to the frontal or temporal lobe by magnetic resonance imaging criteria. Two patients showed soft tissue and/or bone necrosis, and one of these patients required surgical repair of a cerebrospinal fluid leak. The authors concluded that this was a successful treatment approach for these patients. Thornton et al reported encouraging results with treatment of paranasal sinus tumours with combined photon-proton radiotherapy.

At the Loma Linda University in California, 16 patients with recurrent nasopharyngeal carcinoma were treated with conformal proton radiation (Lin et al, 1999). Patients had initially been treated with photon therapy using doses of 50-70 Gy. An additional 59-70 CGE was administered using conformal proton radiation. With a mean follow-up of 23 months, 24-month actuarial overall and local-regional progression-free survival rates were both $50 \%$. No central nervous system complications were observed.

\section{CARCINOMA OF THE PROSTATE}

Photon beam radiation dose escalation was studied in a randomised trial at the MD Anderson Cancer Center (Pollack et al, 2000). For patients with a pretreatment PSA of more than 
$10 \mathrm{ng} \mathrm{ml}^{-1}$, an increase in total dose from 70 to $78 \mathrm{~Gy}$ with conformal photons improved the biochemical disease-free survival. A French trial in which patients were randomised to receive either 70 or $80 \mathrm{~Gy}$ with conformal photons reported no statistical difference in acute toxicity between the two groups; they noted, respectively, 6 and $2 \%$ acute grade 3 urinary and rectal toxicities (Beckendorf et al, 2004). Intensity-modulated radiation therapy has also been used for dose escalation to the prostate while attempting to minimise toxicity by limiting radiation dose to the bladder and rectum. Investigators at the Memorial Sloan Kettering Cancer Center (Zelefsky et al, 2002) treated over 700 patients with IMRT to a dose of at least $81 \mathrm{~Gy}$. Only $28 \%$ of patients experienced grade 2 urinary symptoms and one patient experienced urinary retention. Late grade 2 rectal bleeding was experienced by $1.5 \%$ of patients and four patients required transfusion or laser cauterisation (grade 3). The 3-year actuarial PSA relapse-free survival rates for favourable, intermediate, and unfavourable risk groups were 92,86 , and $81 \%$, respectively.

Investigators at MGH completed a phase III trial comparing 67.2 Gy of photons $v s$ 75.6 CGE using a conformal perineal proton boost for patients with advanced prostate cancer (Shipley et al, 1995). From 1982 through 1992, 202 patients with T3-T4 prostate cancer received $50.4 \mathrm{~Gy}$ by four-field photons. Patients then received either 25.2 CGE with conformal protons or a $16.8 \mathrm{~Gy}$ photon boost. No differences between the two groups were found in overall survival, total recurrence-free survival, or local recurrence-free survival. The local recurrence-free survival at 7 years for patients with poorly differentiated (Gleason 9 and 10) tumours, however, was $85 \%$ on the proton arm and $37 \%$ on the photon arm. Grade 1 and 2 rectal bleeding was higher in the proton arm (32 vs 12\%), as was urethral stricture (19 vs 8\%). In conclusion, dose escalation to 75.6 CGE by conformal proton boost improved local recurrence-free survival in a subset of patients, but also increased late low-grade radiation sequelae; no increase in overall survival was seen in any subgroup.

Investigators at the Loma Linda University Medical Center used proton beam radiotherapy to treat patients with localised prostate cancer. Between 1991 and 1997, over 1200 patients received either all or part of their treatment by proton radiation (Slater et al, 2004). With a median duration of follow-up, overall 5- and 8-year actuarial biochemical disease-free survival rates were 75 and $73 \%$, respectively. Acute grade 3 gastrointestinal and genitourinary (GU) toxicity was less than $1 \%$. Late grade $3 \mathrm{GU}$ toxicity was seen in 14 patients, with eight of them having urethral strictures. The actuarial 5- and 10-year rates for freedom from grade 3 and 4 GU toxicity were both $99 \%$.

MGH and Loma Linda subsequently conducted a phase III randomised trial of radiation dose in patients with early-stage prostate cancer (Zietman et al, 2004). Between 1996 and 1999, 393 patients with early-stage prostate cancer received a conformal proton radiation boost of either 19.8 or 28.8 Gray equivalent (GyE). Following the boost, all patients received 50.4 Gy using 3-D conformal photons to the prostate, seminal vesicles, and periprostatic tissues. At a median follow-up of 4 years, the cumulative incidence estimates of the 5-year local failure rate (using the surrogate of a PSA $>1 \mathrm{ng} \mathrm{ml}^{-1}$ at $>2$ years after radiation were $52.4 \%$ for the $70.2 \mathrm{GyE}$ group and $32.8 \%$ for the $79.2 \mathrm{GyE}$ group $(P<0.001)$. The 5-year biochemical failure rates were $37.3 \%$ for the conventional dose group and $19.1 \%$ for the high-dose group $(P=0.00001)$. These differences were seen for patients with low-risk disease (T1b-2a, PSA $<10$, Gleason $\leqslant 6$ ), as well as intermediate-risk tumours. The 5-year biochemical failure rate in the low-risk group was $34.9 \%$ in the conventional dose arm $v$ s only $17.2 \%$ in the high-dose arm $(P=0.002)$; the figures for intermediate-risk patients were 39.5 vs $21.3 \%(P=0.01)$. At follow-up to date, there was no difference in the overall survival rates between the treatment arms. Importantly, dose escalation was achieved with protons, without any comparable increase in significant acute or late radiation morbidity. Only $2 \%$ of patients receiving conventional dose and $1.5 \%$ receiving high-dose radiation experienced acute urinary or rectal morbidity of RTOG grade $\geqslant 3$. The respective proportions for those experiencing any grade 2 acute morbidity were 62 and $69 \%$. So far only 1.5 and $0.5 \%$, respectively, have experienced late morbidity of RTOG grade $\geqslant 3$.

It will be important to ultimately determine whether proton radiotherapy offers any clinical advantages to patients with prostate cancer compared to IM radiotherapy or (in appropriately selected cases) brachytherapy. This will require randomised clinical trials.

\section{PAEDIATRIC MALIGNANCIES}

Investigators in Switzerland looked at the potential influence of improved dose distribution with proton beams compared to conventional or IM X-ray beams on the incidence of treatmentinduced secondary cancers in children (Miralbell et al, 2002). This model allowed estimation of absolute risks of secondary cancer for each treatment plan based on dose-volume distributions for nontarget organs. Proton beams reduced the expected incidence of radiation-induced secondary cancers for a rhabdomyosarcoma patient by a factor equal to or greater than 2 , and for the medulloblastoma cases a factor of 8-15 (because of the larger target volume) when compared with either IM or conventional Xray plans. This study underscores the concern with using radiation therapy in the treatment of paediatric malignancies. It is the goal of clinicians not only to eradicate the primary tumour but also to minimise the risk of radiation-induced malignancies over the lifetime of these patients. It also underscores the fact that the advantages for protons may be greater with larger rather than smaller target volumes. Owing to technical limitations on field size and depth imposed by some of the modified physic research laboratory facilities employed for treatment in the past, some of the most notable early clinical achievements with protons were with small, superficial targets (i.e. ocular melanomas), leaving the mistaken impression with some clinicians that the advantages for protons were confined to small target volumes.

In a study performed by the MGH group, treatment plans utilising standard photon therapy, IMRT, or protons for craniospinal axis irradiation and posterior fossa boost were compared in a patient with medulloblastoma (St Clair et al, 2004). Substantial normal tissue sparing was realised with IMRT and proton irradiation of the posterior fossa and spinal axis. The dose to $90 \%$ of the cochlea was reduced from $101 \%$ of the prescribed posterior fossa boost dose from conventional X-rays to $33 \%$ with IMRT and to $2 \%$ with protons. Dose to $50 \%$ of the heart volume was reduced from $72 \%$ for photons to $30 \%$ for IMRT and to $0.5 \%$ for protons. (The dose distribution for a child with medulloblastomas undergoing craniospinal irradiation with protons is shown in Figure 2.)

LLU investigators reported a reduction in acute toxicity with the treatment of three children with medulloblastoma treated with craniospinal irradiation using the proton beam technique (Yuh et al, 2004). Loma Linda investigators also evaluated proton beam irradiation in the treatment of paediatric patients with intracranial low-grade astrocytoma (Hug et al, 2002a). Between 1991 and 1997, 27 patients underwent fractionated proton radiation therapy for progression of recurrent low-grade astrocytoma. In all, 25 of the 27 patients $(92 \%)$ were treated for progressive, unresectable, or residual disease following subtotal resection. Mean target dose was 55.2 CGE (50.4-63.0) and fraction size was 1.8 CGE. At a mean follow-up period of 3.3 years ( $0-6.8$ years), six out of 27 patients experienced local failure within the irradiated field and four out of 27 had died. Local control and survival were 87 and 93\%, respectively, for centrally located tumours, 71 and $86 \%$ for hemispheric tumours, and 60 and $60 \%$ for tumours of the 


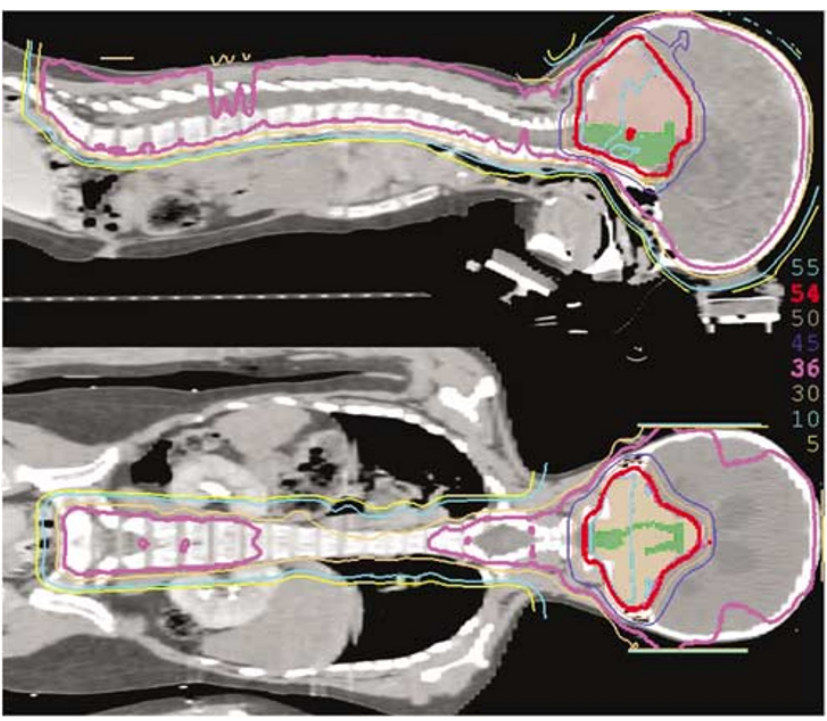

Figure 2 Sagittal and coronal composite dose displays for a child with high-risk medulloblastoma undergoing craniospinal irradiation with protons. Prescription dose to the craniospinal axis for this child with high-risk disease is 36 CGE and the dose to the posterior fossa is 54 CGE. Note the absence of significant exit dose beyond the anterior border of the vertebral bodies, thus sparing the bowel, heart, and mediastinum from potential side effects of radiotherapy.

brainstem. All children with local control maintained their performance status, except one, who developed Moyamoya disease. All six patients with optic pathway tumours and useful vision maintained or improved their visual status.

Four paediatric patients presenting with aggressive giant cell tumours of the skull base were treated with a combination of proton and photon beam radiation at MGH (Hug et al, 2002b). Combined proton and photon radiation therapy was based on 3-D planning. Target doses of 57.6-61.2 CGE were given in daily fractions of 1.8 CGE. With observation times between 3.1 and 5.8 years, all four patients were alive and well and remained locally controlled without evidence of recurrent disease. Except for one patient with partial pituitary insufficiency following radiotherapy for recurrent sellar disease, no late effects attributable to radiation therapy to date have been observed.

Protons offered a preferable dose distribution to photons in two patients treated for orbital rhabdomyosarcoma (Hug et al, 2001). Dose-volume histograms were obtained for target and nontarget regions, including the lens, bony orbit, pituitary gland, optic chiasm, optic nerves, lacrimal gland, and ipsilateral frontal and temporal lobes. Doses to 90,50 , and $5 \%$ of lens volume were kept at less than $1 \%$, less than $2 \%$, and less than $8 \%$, respectively. At a mean follow-up of 3 years, visual acuity for both patients was excellent and there was no evidence of cataract formation. Furthermore, pituitary function was normal; cosmetically, only mild enopthalmos was noticeable. The steep dose gradient beyond the orbit minimised irradiation of normal brain parenchyma, with almost sparing of the pituitary gland.

Ongoing clinical trials of proton beam radiation therapy are in progress at the Northeast Proton Therapy Center (MGH) for paediatric patients with medulloblastoma, rhabdomyosarcoma, other paediatric sarcomas, and retinoblastoma. Protons are also used for treatment of paediatric malignancies at the Loma Linda University Proton Center, and the groups at Orsay in Paris and Paul Scherrer Institute are using proton radiotherapy for paediatric tumours. Protons are also approved for use in patients undergoing radiation therapy as part of treatment on Children's Oncology Group protocols.

\section{OTHER TUMOURS}

Encouraging results with proton beam radiotherapy have also been reported for hepatocellular carcinoma (Chiba et al, 2005) and medically inoperable, early-stage lung cancers (Bush et al, 2004).

\section{CONCLUSIONS}

As discussed, the main benefit of proton therapy over photon beam radiotherapy is the absence of exit dose, which offers the opportunity for highly conformal dose distributions, while simultaneously irradiating less normal tissue. This technology therefore reduces irradiation to normal tissue, while permitting dose escalation to levels not achievable with standard techniques. Dose escalation with protons has been shown in a randomised clinical trial for prostate cancer to improve local tumour control; clinical experience with proton radiotherapy in phase II studies in other anatomic locations suggests that dose escalation in other sites results in improved local control. With reduction of normal tissue dose, proton therapy has been shown to allow for better acute tolerance of combined chemotherapy and radiation therapy; this has been reported for medulloblastoma (Yuh et al, 2004). Ongoing clinical studies are expected to demonstrate similar gains with other tumour types. Improvements in acute tolerance can be expected to minimise interruptions in both chemotherapy and radiotherapy in patients receiving combined modality treatment, with the potential for simultaneous improvement in local and systemic treatment. Equally important is the potential for a decrease in the appearance of late normal tissue effects including radiation-induced malignancies. The importance of this issue cannot be overemphasised when considering the irradiation of paediatric patients.

As noted above, there is also clinical interest in heavier ions, carbon in particular, which have a similar finite range in tissue as protons, a very sharp lateral penumbra, but also have a higher RBE than protons. There is some encouraging preliminary experience from the facility in Chiba and from GSI in Germany (Schulz-Ertner et al, 2004; Tsujii et al, 2004). Further follow-up on these patients, in particular for potential late effects with the higher RBE particles, will be needed before their potential role with respect to protons and IMRT photons can be fully assessed. Carbon ion facilities, however, because of the 12-fold heavier mass of carbon ions compared to protons, are currently more costly than proton facilities, so that appropriate indications will need to be defined.

The expense of proton therapy per patient is expected to decrease as more facilities are built and greater numbers of patients are treated. Current estimates place the relative cost of proton radiation therapy compared to IM photon beam radiation therapy in the range of 2.4, but might come down to 1.7-2.1 over the next 5 years (Goitein and Jermann, 2003). A recent publication from Sweden actually projected lower health-care expenses using proton beam radiotherapy when compared to conventional radiation therapy in the treatment of a child with medulloblastoma, because of the substantial health-care burden in managing the late effects of conventional radiotherapy (Lundkvist et al, 2005).

At the present time, we believe that all paediatric patients should be considered for referral, as well as all cases where the proximity of tumour to critical structures prohibits the administration of adequate radiation doses using photon techniques. Rapid advances in photon radiotherapy with image-guided IMRT, stereotactic radiotherapy, and brachytherpy are, however, competing technologies for adult patients and appropriate clinical studies will be important to define the relative benefits and indications for these different technologies. 


\section{REFERENCES}

Beckendorf V, Guerif S, Prise E (2004) The GETUG 70 vs 80 Gy randomized trial for localized prostate cancer: feasibility and acute toxicity. Int J Radiat Oncol Biol Phys 60: 1056-1065

Bush DA, Slater JD, Shin BB, Cheek G, Miller DW, Slater JM (2004) Hypfractionated proton beam radiotherapy for stage I lung cancer. Chest 126: $1198-1203$

Chiba T, Tokuuye K, Matsuzaki Y, Sugahara S, Chuganji Y, Kagei K, SHoda J, Hata M, Abei M, Igaki H, Tanaka N, Akine Y (2005) Proton beam therapy for hepatocellular carcinoma: a retrospective review of 162 patients. Clin Cancer Res 11: 3799-3805

Egger E, Zografos L, Schalenbourg A, Beati D, Bohringer T, Chamot L, Goitein G (2003) Eye retention after proton beam radiotherapy for uveal melanoma. Int J Radiat Oncol Biol Phys 55: 867-880

Fitzek MM, Thornton AF, Varvares M (2002) Neuroendocrine tumors of the sinonasal tract. Results of a prospective study incorporating chemotherapy, surgery, and combined proton-photon radiotherapy. Cancer 94: $2623-2634$

Goitein M, Jermann M (2003) The relative costs of proton and X-ray radiation therapy. Clin Oncol ( $R$ Coll Radiol) 15: S37-S50

Hug EB, Fitzek MM, Liebsch NJ (1995) Locally challenging osteo- and chondrogenic tumors of the axial skeleton: results of combined proton and photon radiation therapy using three-dimensional treatment planning. Int J Radiat Oncol Biol Phys 31(3): 467

Hug EB, Muenter MW, Adams JA, de Vries A, Rosenberg AE, Munzenrider JE (2002b) 3-D-conformal radiation therapy for pediatric giant cell tumors of the skull base. Strahlenther Onkol 178: 239-244

Hug EB, Muenter MW, Archambeau JO (2002a) Conformal proton radiation therapy for pediatric low-grade astrocytomas. Strahlenther Onkol 178: $10-17$

Hug EB, Nevinny-Stickel M, Fuss M, Miller DW, Schaefer RA, Slater JD (2001) Conformal proton radiation treatment for retroperitoneal neuroblastoma: introduction of a novel technique. Med Pediatr Oncol 37: $36-41$

Kamada T, Tsujii H, Tsuji H (2002) Efficacy and safety of carbon ion radiotherapy in bone and soft tissue sarcomas. J Clin Oncol 20: $4466-4471$

Lin R, Slater JD, Yonemoto LT (1999) Nasopharyngeal carcinoma: repeat treatment with conformal proton therapy - dose-volume histogram analysis. Radiology 213: 489-494

Lundkvist J, Ekman M, Ericcson SR, Jonsson B, Glimelius B (2005) Cost effectiveness of proton radiation in the treatment of childhood medulloblastoma. Cancer 103: 793-801

Miralbell R, Lomax A, Cella L, Schneider U (2002) Potential reduction of the incidence of radiation-induced second cancers by using proton beams in the treatment of pediatric tumors. Int J Radiat Oncol Biol Phys 54: $824-829$

Munzenrider JE (1999) Proton therapy for uveal melanomas and other eye lesions. Srahlenther Onkol 175: 68

Munzenrider JE, Liebsch NJ (1999) Proton therapy for tumors of the skull base. Strahlenther Onkol 175: 57-63

Paganetti H, Nimierko A, Ancukiewicz M, Gerweck LE, Goitein M, Loeffler JS, Suit HD (2002) Relative biological effectiveness (RBE) values for proton beam radiotherapy. Int J Radiat Oncol Biol Phys 53: $407-421$

Pai HH, Thornton A, Katznelson L (2001) Hypothalamic/pituitary function following high-dose conformal radiotherapy to the base of skull: demonstration of a dose-effect relationship using dose-volume histogram analysis. Int J Radiat Oncol Biol Phys 49: 1079-1092
Pollack A, Zagars G, Smith L (2000) Preliminary results of a randomized radiotherapy dose-escalation study comparing $70 \mathrm{~Gy}$ with $78 \mathrm{~Gy}$ for prostate cancer. J Clin Oncol 18: 3904-3911

Schulz-Ertner D, Nikoghosyan A, Thilmann C, Haberer T, Jakel O, Karger C, Kraft G, Wannenmacher M, Debus J (2004) Results of carbon ion radiotherapy in 152 patients. Int J Radiat Oncol Biol Phys 58: 631-640

Shipley WU, Verhey LJ, Munzenrider JE (1995) Advanced prostate cancer: the results of a randomized comparative trial of high dose irradiation boosting with conformal protons compared with conventional dose irradiation using photons alone. Int J Radiat Oncol Biol Phys 32: 3-12

Sisterson J (2004) World wide charged particle patient totals. Particles 30: 20

St Clair WH, Adams JA, Bues M (2004) Advantage of protons compared to conventional X-ray or IMRT in the treatment of a pediatric patient with medulloblastoma. Int J Radiat Oncol Biol Phys 58: 727-734

Slater JD, Rossi Jr CJ, Yonemoto LT, Bush DA, Jabola BR, Levy RP, Grove RI, Preston W, Slater JM (2004) Proton therapy for prostate cancer: the initial Loma Linda University experience. Int J Radiat Oncol Biol Phys 59: $348-352$

Thornton AF, Fitzek MM, Varvarres M (1998) Accelerated, hyperfractionated photon/proton irradiation for advanced paranasal sinus cancer: results of a prospective phase I-II study. Int J Radiat Oncol Biol Phys 42: 222

Tsujii H, Mizoe JE, Kamada T, Baba M, Kato S, Kato H, Tsuji H, Yamada S, Yasuda S, Ohno T, Yanagi T, Hasegawa A, Sugawara T, Erzawa H, Kandatsu S, Yoshikawa K, Kishimoto R, Miyamoto T (2004) Overview of clinical experiences on carbon ion therapy at NIRS. Radiother Oncol 73(Suppl 2): S41 - S49

Uy NW, Woo SY, Mai WY (2002) Intensity modulated radiation therapy for meningioma. Int J Radiat Oncol Biol Phys 53: 1265-1270

Wambersie A, Menzel HG (1996) Present status, trends, and needs in fast neutron therapy. Bull Cancer Radiother 83(Suppl): 68s-77s

Weber DC, Lomax AJ, Rutz HP (2004) Spot-scanning proton radiation therapy for recurrent, residual or untreated intracranial meningiomas. Radiother Oncol 71: 251-258

Wenkel E, Thornton AF, Finkelstein D (2000) Benign meningioma: partially resected, biopsied, and recurrent intracranial tumors treated with combined proton and photon radiotherapy. Int J Radiat Oncol Biol Phys 48: $1363-1370$

Wilson RR (1946) Radiological uses of fast protons. Radiology 47: 487

Yuh GE, Loredo LN, Yonemoto LT, Bush DA, Shahnazi K, Preston W, Slater JM, Slater JD (2004) Reducing toxicity from craniospinal irradiation: using proton beams to treat medulloblastoma in young children. Cancer J 10: $386-390$

Zabel SM, Zabel A, Ertner DS (2005) Fractionated stereotactic radiotherapy in patients with benign or atypical intracranial meningioma: long-term experience and prognostic factors. Int J Radiat Oncol Biol Phys 61: $809-816$

Zelefsky MJ, Fuks Z, Hunt M, Liebel SA (2002) High dose intensity modulated radiation therapy for prostate cancer: early toxicity and biochemical outcome in 772 patients. Int J Radiat Oncol Biol Phys 53: $111-116$

Zietman AL, DeSilvio M, Slater JD, Rossi CJ, Yonemoto LT, Slater JM, Miller DW, Berkley B, Adams JA, Shipley WU (2004) A randomized trial comparing conventional dose $(70.2 \mathrm{GyE})$ and high-dose $(79.2 \mathrm{GyE})$ conformal radiation in early stage adenocarcinoma of the prostate: results of an interim analysis of PROG 95-09. Int J Radiat Oncol Biol Phys 60: 131 